\title{
General practice out-of-hours service in Ireland provides a new source of syndromic surveillance data on influenza
}

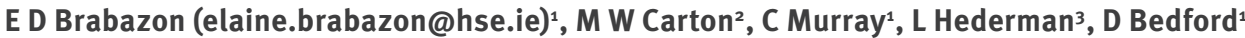

1. Department of Public Health, Health Service Executive Dublin North East, Navan, County Meath, Ireland

2. Infection Prevention \& Control, Cavan Monaghan Hospital Group, Health Service Executive Dublin North East, Cavan, Ireland

3. Department of Computer Science, Trinity College, Dublin, Ireland

Citation style for this article:

Brabazon ED, Carton MW, Murray C, Hederman L, Bedford D. General practice out-of-hours service in Ireland provides a new source of syndromic surveillance data on influenza. Euro Surveill. 2010;15(31):pii=19632. Available online: http://www.eurosurveillance.org/ViewArticle.aspx?Articleld=19632

Article published on 5 August 2010

The use of routinely available electronic sources of healthcare data on the spread of influenza has the potential to enhance current surveillance activities. This study aimed to develop a method for identifying influenza-related records from general practitioner (GP) out-of-hours $(\mathrm{OOH})$ services in Ireland. Data from one such service were interrogated for keywords relating to influenza-like illness (ILI) and a proxy measure of influenza activity in the community setting was developed. Comparison of this syndromic surveillance measure with national data on ILI consultation rates demonstrated a statistically significant temporal correlation. In five out of six influenza seasons investigated, peaks in the GP OOH influenza-related calls appeared at least one week ahead of peaks in the national ILI consultation rates. The method described in this paper has been extended to nine $\mathrm{OOH}$ services in Ireland (covering $70 \%$ of the Irish population) to provide weekly figures on self-reported illness for influenza in the community and its data have been incorporated into the national weekly influenza reports produced by the Health Protection Surveillance Centre. These data should provide early warnings of both seasonal and pandemic influenza in Ireland.

\section{Introduction}

The recent influenza pandemic reemphasised the need to ensure that a reliable, comprehensive system is in place for influenza surveillance. It is worthwhile assessing all available data sources for their potential for detecting influenza. Indicators of influenza activity in the community warrant particular attention. Data that are gathered electronically and maintained as part of routine community medical care might be expected to provide a timely and readily available source of information on the transmission of influenza and influenzalike illness (ILI).

\section{Telehealth data in Ireland}

Data from healthcare call centres or 'telehealth' data, which log direct interactions of patients with a healthcare service and maintain records of patients' symptoms at particular points in time, have in recent years become a source of information for syndromic surveillance. Systems such as NHS Direct in the United Kingdom [1-5], the Ontario Telehealth System in Canada $[6,7]$ and Melbourne Medical Deputising Service in Australia [8] have been used successfully to monitor trends in ILI.

In Ireland, one such telehealth system is the out-ofhours $(\mathrm{OOH})$ or 'doctor-on-call' service, which provides general practitioner (GP) care at times when doctors' surgeries are closed. Since 1998, nine OOH cooperative organisations (coops) have been developed, which cover about $70 \%$ of the Irish population [9]. These services operate from 18:00 to 08:00 on weekdays and 24 hours a day during weekends and bank holidays. The mean contact rate is 221 contacts per 1,000 population per year [10]. These cooperatives aim to provide an urgent $\mathrm{OOH}$ GP service to their patients and to facilitate continuity of care. The services provide access to a doctor through a call centre telephone number. Clerical personnel answer calls and record details in a computerised system. The information is triaged by either a nurse or a doctor (depending on the $\mathrm{OOH}$ service) and a clinician calls the patient back promptly. The doctor may offer advice on the telephone or arrange for the patient to be seen at the local doctor-on-call centre or visited at home if necessary. Details of the consultation are recorded in the computer system and the information is faxed to the patient's own GP before surgery reopens, ensuring that follow-up can be carried out if required.

Large data repositories, which contain records of selfreported illness (syndromes) in the community and clinicians' subsequent diagnoses, are therefore maintained by the GP OOH service. The potential exists to utilise this valuable data source as an early warning or alert system for community illness in Ireland. 


\section{National sentinel GP influenza}

surveillance system

The Irish sentinel GP influenza surveillance system gathers data electronically from sentinel GPs throughout the country to provide clinical data on all cases of ILI diagnosed in their practices each week. These data form the basis for the main national influenza indicator, the GP ILI consultation rates, and provide internationally comparable figures. During the 2008-9 influenza season, 54 GPs took part in the surveillance programme, which covered about $5 \%$ of the Irish population. Sentinel GPs are also required to send nasal and throat swabs - for at least one patient per week presenting with ILI - to the National Virus Reference Laboratory for virological type confirmation. Other sentinel data from hospitals and schools around the country are also monitored nationally during the influenza season (further information on routine national influenza surveillance can be found at http://www. hpsc.ie/hpsc/A-Z/Respiratory/Influenza/).

The aim of this work was to identify a feasible and practical means for identifying ILI-related records from $\mathrm{OOH}$ services in Ireland and to evaluate the results by comparing this syndromic surveillance data with national indicators and standards for influenza. To the best of our knowledge, this is the first time in Ireland that GP $\mathrm{OOH}$ services have been investigated as a potential source of syndromic surveillance data.

\section{Methods}

\section{Strategy}

Calls to GP OOH services in Ireland are not currently recorded using clinical codes, so a strategy to identify influenza-related calls had to be developed. We searched for specific key words, using Microsoft (MS) Access [11] to interrogate the data. The aim of this process was to design and validate a query that would act as a proxy measure for influenza activity in the community.

\section{Quantitative data analysis}

Anonymised data on all calls for six influenza seasons between 2003 and 2009 were obtained from one call centre that covers two $\mathrm{OOH}$ GP services (North East Doctor on Call and Midlands Doctor on Call). Data were extracted from the Adastra software system [12] into plain text or comma separated values (CSV) files. The fields in the datasets consisted of call number, date of call, patient age, patient sex, patient's reported condition, and doctor's diagnosis/outcome. Records that contained 'test call' in the reported condition field were deleted from the file - these referred to information technology maintenance records. Data were imported into MS Access to enable us to examine freetext fields. Data were aggregated weekly (Monday to Sunday inclusive) to coincide with the weekly influenza season calendar, which runs from week 40 (mid-September) to week 20 (mid-May).
Design of queries

Comparing total calls per week with the national ILI consultation rates for the corresponding periods showed no particular synchronisation (Spearman's rank correlation coefficient ranged from -0.02 to 0.42 over the six years investigated). Therefore, in an attempt to identify specific influenza/ILI-related records in the dataset, keyword queries were designed in MS Access to extract records based on information from the 'patient's reported condition' field as this field contained a large amount of information for each record. Three queries were initially designed. The first was based on the Irish national definition for ILI used by the Health Protection Surveillance Centre (HPSC) [13], the second on an American definition for ILI used by the United States Centers for Disease Control and Prevention (CDC) [14] and the third on a self-reported illness query for flu/influenza. The query for the national ILI standard definition was designed to extract records with the terms 'fever' or 'high temperature' and two or more of the following - 'headache', 'sore throat', 'cough' and 'aches and pains' ('aches and pains' was chosen instead of 'myalgia' in the national ILI definition as it represents the lay term for the medical phrase). The query for CDC's ILI definition was designed to extract records with the terms 'fever' or 'high temperature' and 'cough' or 'sore throat'. The final query, based on patients' self-reported illness, was designed to extract the keywords 'flu' or 'influenza'.

Choice of proxy measure for influenza activity In order to ensure that the queries extracted appropriate records from the free-text fields - in other words to test, refine and validate these specific queries - a subset of data $(5,732$ records from a period of high influenza activity during the 2005-6 season) were manually categorised using a binary system. For all 5,732 records, the value of 1 or o was allocated for each of a range of influenza-related keywords recorded in the patient's reported condition field (i.e. headache, cough, sore throat, high temperature, aches and pains, fever, flu, influenza). Specific binary codes represented the national ILI definition, the CDC definition, or the self-reported illness definition for influenza and each

\section{TABLE 1}

Sensitivity, specificity and positive predictive values for three query methods, Ireland, 2005-6 influenza season $(n=5,732)$

\begin{tabular}{|l|c|c|c|}
\hline Query & $\begin{array}{c}\text { Sensitivity } \\
(\%)\end{array}$ & $\begin{array}{c}\text { Specificity } \\
(\%)\end{array}$ & $\begin{array}{c}\text { Positive } \\
\text { predictive } \\
\text { value (\%) }\end{array}$ \\
\hline $\begin{array}{l}\text { Irish national ILI } \\
\text { definition }\end{array}$ & 97.7 & 99.9 & 93.6 \\
\hline CDC ILI definition & 98.6 & 100 & 100 \\
\hline $\begin{array}{l}\text { Flu/influenza self- } \\
\text { reported illness definition }\end{array}$ & 100 & 99.9 & 98.6 \\
\hline
\end{tabular}

CDC: United States Centers for Disease Control and Prevention; ILI: influenza-like illness. 
record was classified accordingly. Thus it was possible to compare the manually categorised subset of data with the results of the MS Access queries. For each query, true positives, true negatives, false positives and false negatives were identified, and sensitivity, specificity and positive predictive values were calculated for each individual query. All queries had high sensitivity, specificity, and positive predictive values ( $293 \%$; Table 1), but these values were lowest for the query for the national definition for ILI. The complex nature of the national ILI case definition means that inevitability a slightly higher level of false positives will be extracted from a free-text system.

Comparison of the data extracted for the three queries over the six influenza seasons showed that the numbers of records extracted overall for the national definition query were much lower than for the other queries (overall only $0.37 \%$ of all calls were extracted with this query) and therefore the data were quite 'noisy' and uninformative (Figure 1 as an example). For the query representing the American ILI definition during the six influenza seasons, peaks occurred during the periods of increased national consultation rates for ILI and more records were extracted than for the national ILI definition. The highest numbers of records, however, were extracted with the self-reported illness query for flu/influenza (overall $3.2 \%$ of all calls were extracted with this query). This trend was seen consistently in all the influenza seasons we investigated and substantial peaks were obtained throughout the six-year period for the self-reported illness query. The self-reported illness query was used, therefore, as our proxy measure of influenza activity in the community.

\section{Source of data on routine influenza surveillance indicators}

National data on routine influenza surveillance indicators, including GP ILI consultation rates, were obtained upon request from the HPSC.

\section{Statistical analysis}

Data were analysed using the statistics package JMP [15]. We used Spearman's rank correlation coefficient to compare temporal trends in the extracted $\mathrm{OOH}$ data and national influenza indicators. This tool has been used previously $[7,16,17]$ to assess the relationship between syndromic influenza surveillance datasets and corresponding influenza indicators.

\section{Results}

\section{All calls data}

In total, during the six influenza seasons between 2003-4 and 2008-9, there were 539,732 calls to the two GP OOH services studied (Table 2), a mean of 2,712 calls per week. These calls included $\mathbf{2 1 0 , 9 3 2}$ calls $(39 \%)$ for paediatric patients ( 15 years of age), 253,703 calls $(47 \%)$ for patients aged 15 to 69 years, and 73,648 calls $(14 \%)$ for patients aged 70 years of age and over. More calls were received for female patients than for male patients (male to female ratio 1:1.2). Calls to the services increased year by year, as more doctors joined the scheme (199 GPs in 2003,

\section{FIGURE 1}

Ascertainment of apparent influenza using three query methods, Ireland, 2003-4 influenza season

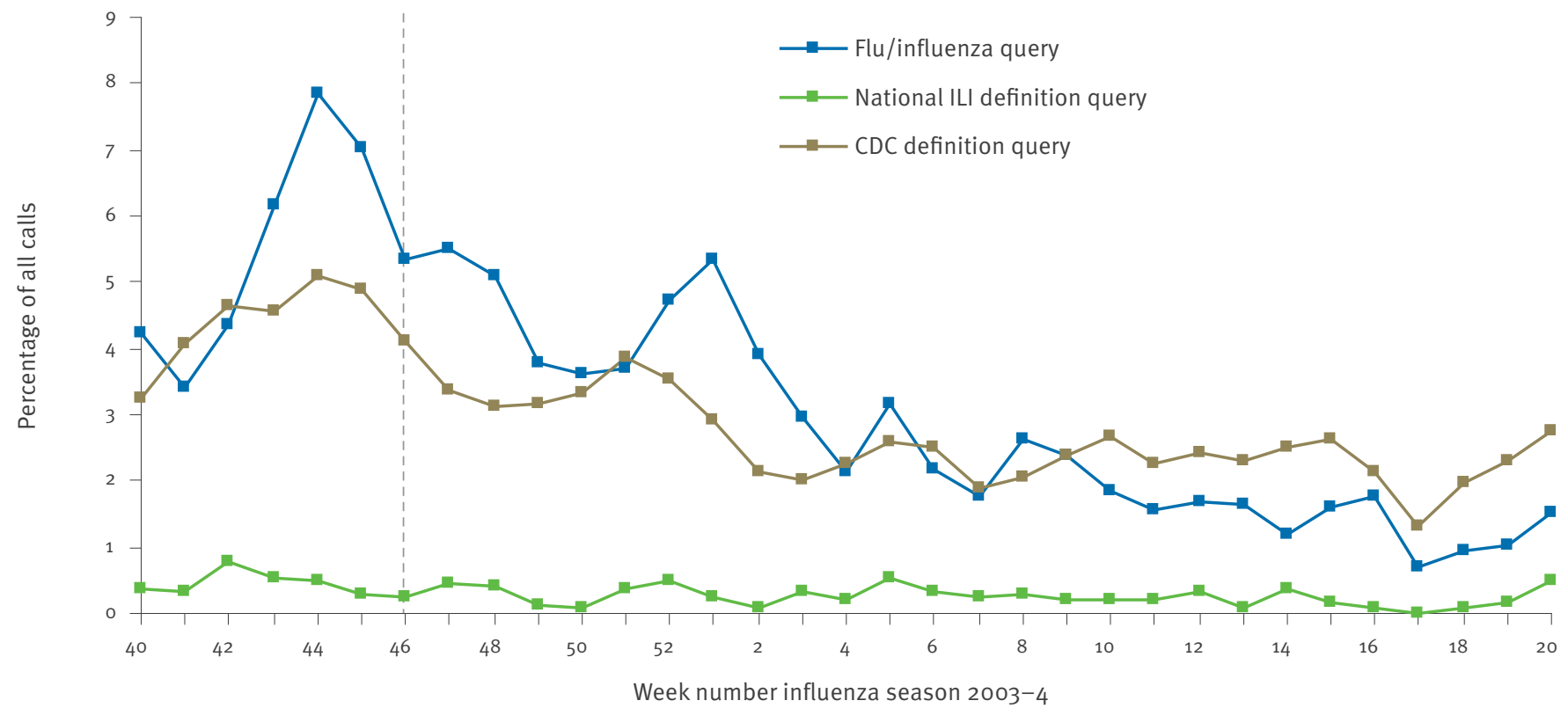

CDC: United States Centers for Disease Control and Prevention; ILI: influenza-like illness.

General practice out-of-office hours extracted data are plotted as a weekly proportion of all calls. The vertical dashed line highlights the week of peak national ILI consultations. 
$269 \mathrm{GPs}$ in 2009). The population coverage grew from 298,500 to 403,500 between 2003 and 2009 (see [10] for calculation of population coverage estimates for Irish GP OOH coops). Between 2003 and 2009 these GP coops provided $\mathrm{OOH}$ care for between $7 \%$ and $9.5 \%$ of the national population.

\section{Ascertainment of influenza-like illness from} general practitioner out-of-office hours records Over the six influenza seasons, 17,062 records (3.2\% of all records) were extracted using the self-reported illness query for flu/influenza (Table 2). The commonest age group for these calls varied with the influenza season. Calls were commonest among those aged 70 years and over for the first four seasons (2003-4 to 2006-7), in the 30-34 years group in 2007-8, and in children aged four years and under in 2008-9. Female patients accounted for more calls for flu or influenza symptoms than male patients.

\section{Seasonal comparison with national indicators}

The extracted records and national ILI consultation rates were compared for each influenza season over the six-year period by time series plot (Figure 2). The relationship between the datasets followed a similar pattern each year. National rates for ILI consultations peaked at various points in the season and peaks in extracted call data occurred at much the same time - early in the 2003-4 influenza season; over the Christmas period in the 2004-5, 2007-8, and 2008-9 seasons; and late in the season in 2005-6 and 2006-7 (Table 2 and Figure 2). The most notable difference between the datasets was that the peak in extracted call data occurred before the peak in national ILI consultation rates in five out of the six influenza seasons studied and was on average 1.5 weeks earlier over the six-year period (Table 2). It is worth noting that late in the 2007-8 season, a second peak was observed in the regional ILI rates that was not reflected in the national data. A corresponding second peak was also observed in the call data (Figure 2). This demonstrates the ability of the call data to identify regional trends in influenza activity.

Spearman's rank correlation coefficient demonstrated a statistically significant temporal correlation between extracted call data and national ILI rates for all six seasons (Table 2). The highest correlation (0.909) was seen for the 2003-4 season, when two distinct peaks in activity early in the season were evident in both datasets. The lowest correlation (although still statistically significant) was observed in 2005-6.

\section{Discussion}

The GP OOH services in Ireland account for substantial healthcare interactions with patients in the community, and details of its activity are routinely recorded on computer. We have validated a method for extracting influenza-related call data from one such service using a commonly available database software package. Specific keyword queries were designed, based on widely accepted definitions for ILI. This was necessary as the service maintains records of the patients self-reported symptoms or illness in free text, rather than a clinical coding system.

We have shown from the six-year period examined in this study that extracted influenza-related call data closely matched patterns in national ILI rates (our gold standard) and this temporal association was shown to be statistically significant. For five out of the six seasons analysed, the week with the highest number of influenza-related calls for each season was at least one week ahead of the peaks in national ILI rates,

\section{TABLE 2}

Influenza-related calls extracted from general practitioner out-of-office hours data using influenza proxy measure, 2003-4 to $2008-9$ influenza seasons

\begin{tabular}{|c|c|c|c|c|c|c|}
\hline \multirow{2}{*}{ Item } & \multicolumn{6}{|c|}{ Influenza season } \\
\hline & $2003-4$ & $2004-5$ & $2005-6$ & $2006-7$ & $2007-8$ & $2008-9$ \\
\hline Total number of call records for season & 71,703 & 83,269 & 84,764 & 94,638 & 103,191 & 102,167 \\
\hline Number of call records extracted (\%) & $2,376(3.3)$ & $3,004(3.6)$ & $2,308(2.7)$ & $2,629(2.8)$ & $3,275(3.2)$ & $3,470(3.4)$ \\
\hline $\begin{array}{l}\text { Male:female ratio for patients, from } \\
\text { extracted records }\end{array}$ & 1:1.4 & $1: 1.2$ & $1: 1.3$ & 1:1.3 & $1: 1.2$ & 1:1.4 \\
\hline $\begin{array}{l}\text { Modal age group of patients } \\
\text { (number of extracted records) }\end{array}$ & $\begin{array}{c}\geq 70 \text { years } \\
(295)\end{array}$ & $\begin{array}{l}\geq 70 \text { years } \\
(292)\end{array}$ & $\begin{array}{c}\geq 70 \text { years } \\
(238)\end{array}$ & $\begin{array}{l}\geq 70 \text { years } \\
(283)\end{array}$ & $\begin{array}{c}\text { 30-34 years } \\
(343)\end{array}$ & $\begin{array}{l}0-4 \text { years } \\
(363)\end{array}$ \\
\hline $\begin{array}{l}\text { Week of highest number of extracted } \\
\text { records/total (percentage of total) }\end{array}$ & W44 (7.85) & $W_{53}(11.1)$ & W6 (4.9) & W6 (6) & $W_{1}(7.8)$ & $W_{52}(19)$ \\
\hline Week of peak national ILI consultations & W46 & $\mathrm{W}_{1}$ & $W_{10}$ & $\mathrm{w}_{7}$ & $\mathrm{~W}_{1}$ & $\mathrm{~W}_{2}$ \\
\hline $\begin{array}{l}\text { Number of weeks call-data peak leads ILI- } \\
\text { data peak }\end{array}$ & 2 & 1 & 4 & 1 & 0 & 2 \\
\hline Spearman's rank correlation coefficient ${ }^{\mathrm{a}}$ & 0.901 & 0.709 & 0.679 & 0.888 & 0.858 & 0.759 \\
\hline
\end{tabular}

ILI: influenza-like illness; W: week.

a Spearman's rank correlation coefficient was calculated between the extracted calls as a proportion of total calls and the national ILI consultation rates compared weekly during the corresponding influenza season. 
thus offering the potential for our self-reported illness query to be a useful syndromic surveillance measure, an early warning system.

The $\mathrm{OOH}$ service may provide an earlier indication of ILI activity partly because of the long periods for which it is operational. During an average week, the $\mathrm{OOH}$ service is in operation for over two thirds of the week (118 of 168 hours) and therefore patients with acute illnesses that need urgent attention may contact the $\mathrm{OOH}$ service rather than wait to make a daytime appointment to see their GP.

GP coops that provide $\mathrm{OOH}$ healthcare have become widely used by the Irish population in recent years $[9,10]$. The large scale of this interaction with the population and electronic availability of their records are not the only advantages that $\mathrm{OOH}$ datasets can provide. Data are entered into computer systems in real time while a patient calls the service, which means that information about the patient's reported condition, and often the doctor's diagnosis, is potentially available immediately for analysis. This may have important implications during a pandemic, when trends in influenza transmission may need to be analysed daily. Other than the information technology requirement to extract information daily or weekly, depending on the surveillance protocol, the service would bear no additional costs or workload, as the data would be collected routinely in any case.

Another aspect of the dataset which could be utilised is the linkage of patient demographic data to the call record. Although this information was not collected for analysis in this study due to data protection issues, it may be possible in the future to map demographic data for influenza-related calls by time and location. This type of analysis has the potential to identify local influenza outbreaks and their points of origin, thereby providing valuable insights on the geographical dissemination of new influenza viruses. Analysis of this type has already been employed with influenza military emergency and primary care surveillance data in the United States [16] and fever and vomiting calls to NHS Direct in the United Kingdom [3] to generate a 'moving picture' or tracking of cases through location and time.

There are, of course, some caveats to using data from the $\mathrm{OOH}$ services as an indicator of influenza activity. In the first instance, these services operate outside normal GP working hours (i.e. 18:00 to 08:00 for Monday to Friday and 24 hours for weekends and bank holidays). This means that some patients with ILI who present at GP surgeries during the day are being lost to this surveillance process. In addition, although most GPs in Ireland do participate in these $\mathrm{OOH}$ coops, the service is not ubiquitous. As the scheme is voluntary among GPs and GP practices are independent businesses, the services may never reach $100 \%$ coverage of the Irish population. The most important limitation of the data from the $\mathrm{OOH}$ services is the recording of the patient's reported condition in a free-text format. Clearly, analysis of free-text fields to identify trends and patterns in data over time can be a time-consuming and difficult task. Other analyses of influenzarelated healthcare syndromic surveillance data have used software systems that map symptoms to syndromes [18-22] or used a decision support system or natural language processing tool to categorise all calls $[1,2,17,23,24]$.

\section{FIGURE 2}

National data on influenza-like illness consultation rate ${ }^{\mathrm{a}}$ and extracted influenza calls ${ }^{\mathrm{b}}, 2003-4$ to $2008-9$ influenza seasons

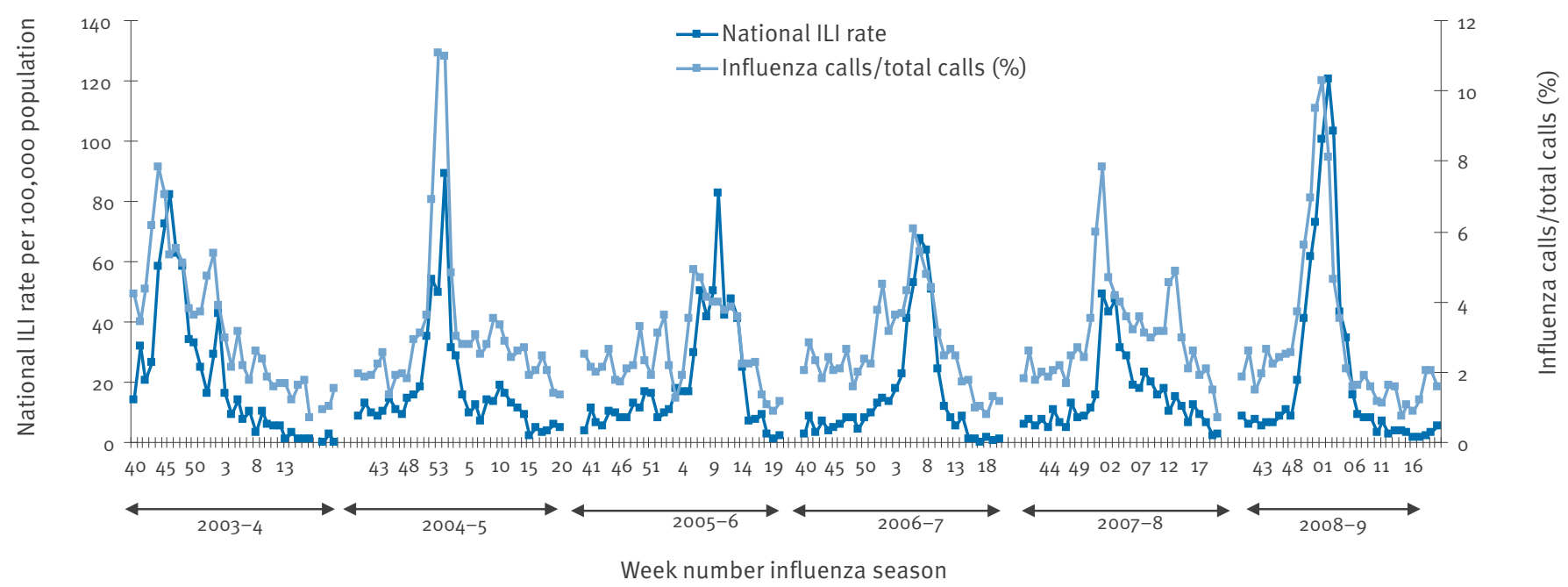

ILI: influenza-like illness.

a Per 100,000 population per week.

b As a percentage of all weekly calls to the general practitioner out-of-office hours services under study. 
Currently, there is no facility for mapping of patient symptoms to syndromes or clinical coding - e.g. International Classification of Diseases (ICD) - of the data by the majority of Irish $\mathrm{OOH}$ services, although the Adastra software system could be upgraded to allow for this. The benefits of such an upgrade would include uniform classification of calls, the ability to easily review and analyse trends over time and the possibility of integration into a syndromic surveillance system that could provide an early warning system for outbreaks and other conditions of public health importance. However, additional time on each call and a change in work practice by the services would be needed in order to implement coding/mapping on the service and this is not envisaged at present.

On completion of this study, the nine $\mathrm{OOH}$ services in Ireland were approached and asked to provide a weekly extract for analysis of influenza-related calls using the methodology described in this paper. All of these $\mathrm{OOH}$ services now provide data regularly for analysis, covering about $70 \%$ (three million) of the Irish population. Results of this weekly analysis have been available since week 19 of the 2008-9 influenza season and are included in the national weekly influenza report produced by the HPSC [13]. Increases in influenza-related calls to these $\mathrm{OOH}$ services observed since week 29 of the season coincided with increases seen in national ILI consultation rates, reflecting the spread of the pandemic influenza $\mathrm{A}\left(\mathrm{H}_{1} \mathrm{~N}_{1}\right)$ virus in Ireland.

Most significantly, the weekly analysis of the GP OOH influenza-related call data was used by the National Crisis Management Team in combination with other influenza indicators to shape the response to the 2009 influenza pandemic in Ireland. These data may also provide information for action at local level. It has been observed anecdotally that increases in calls for influenza in younger age groups to the $\mathrm{OOH}$ service may herald an increase in presentations to hospital emergency departments. Advance notice of such increases in the community, particularly during a pandemic, can give hospitals valuable time to prepare separate waiting areas, obtain adequate stocks of antiviral drugs and diagnostic swabs, and ensure that an action plan for rapid admission to isolation of suspected cases is in place. Furthermore, analysis of past trends in influenza-related calls can allow individual $\mathrm{OOH}$ services to review their own personnel arrangements for peak influenza and indeed pandemic periods.

\section{Conclusions}

This study has shown that it is possible to extract influenza-related patient contact records from an urgent $\mathrm{OOH}$ GP service in Ireland and that the data have the potential to be utilised as an early alert system for seasonal and pandemic influenza. Collection of data from all $\mathrm{OOH}$ services to obtain a national overview can provide valuable epidemiological information at a relatively low resource and infrastructure cost. This meth- odology could be applied to other important areas of public health surveillance.

\section{Acknowledgements}

The authors would like to thank the GP OOH services for their continuing participation in this study and also the HPSC for providing the national influenza indicator data.

References

1. Harcourt SE, Smith GE, Hollyoak VA, Joseph CA, Chaloner R, Rehman Y, et al. Can calls to NHS-Direct be used for syndromic surveillance? Commun Dis Public Health. 2001;4(3):178-82.

2. Cooper DL, Smith GE, Hollyoak VA, Joseph CA, Johnson L, Chaloner R. Use of NHS Direct calls for surveillance of influenza--a second year's experience. Commun Dis Public Health. 2002;5 (2):127-31.

3. Cooper DL, Smith GE, Regan M, Large S, Groenewegen P. Tracking the spatial diffusion of influenza and norovirus using telehealth data: a spatiotemporal analysis of syndromic data. BMC Med. 2008;6:16.

4. Cooper DL, Verlander NQ, Elliott AJ, Joseph CA, Smith GE. Can syndromic thresholds provide early warning of national influenza outbreaks? J Public Health (Oxf). 2007;31(1):17-25.

5. Smith GE, Cooper DL, Loveridge P, Chinemana F, Gerard E, Verlande N. A national syndromic surveillance system for England and Wales using calls to a telephone helpline. Euro Surveill. 2006;11(12). pii: 667. Available from: http://www. eurosurveillance.org/ViewArticle.aspx?Articleld $=667$

6. Rolland E, Moore KM, Robinson VA, McGuinness D. Using Ontario's "Telehealth" health telephone helpline as an earlywarning system: a study protocol. BMC Health Serv Res. 2006;6:10.

7. van Dijk A, McGuinness D, Rolland E, Moore KM. Can Telehealth Ontario respiratory call volume be used as a proxy for emergency department respiratory visit surveillance by public health? CJEM. 2008;10(1):18-24.

8. Coory M, Grant K, Kelly H. Influenza-like illness surveillance using a deputising medical service corresponds to surveillance from sentinel general practices. Euro Surveill. 2009:14(44). pii: 19387. Available from: http://www.eurosurveillance.org/ ViewArticle.aspx?Articleld $=19387$

9. Health Service Executive (HSE). National review of GP out of hours' services. HSE; 2010. Available from: http://www.lenus. $\mathrm{ie} / \mathrm{hse} / \mathrm{handle} / 10147 / 96875$ ? mode $=$ full

10. Bury G, Dowling J, Janes D. General practice out of hours co-operatives--population contact rates. Ir Med J. 2006;99(3):73-5.

11. Microsoft Corporation. Access 2010. Available from: http:// office.microsoft.com/en-us/access

12. Adastra Software Ltd. (2009) Available from: http://www. adastra.com

13. Health Protection Surveillance Centre (HPSC). Influenza surveillance. Dublin: HPSC; 2009. Available from: http://www. ndsc.ie/hpsc/A-Z/Respiratory/Influenza/Publications/

14. Centers for Disease Prevention and Control (CDC). Flu activity \& surveillance. Reports \& surveillance methods in the United States. Past Weekly Surveillance Reports. Atlanta: CDC; 2009. Available from: http://www.cdc.gov/flu/weekly/fluactivity.htm

15. JMP Statistical Software (SAS Institute Inc). [Home page]. Available from: http://www.jmp.com

16. Lewis MD, Pavlin JA, Mansfield IL, O’Brien S, Boomsma LG, Elbert $Y$, et al. Disease outbreak detection system using syndromic data in the Greater Washington DC area. Am J Prev Med. 2002;23(3):180-6.

17. Doroshenko A, Cooper D, Smith G, Gerard E, Chinemana F, Verlander N, et al. Evaluation of syndromic surveillance based on National Health Service Direct derived data--England and Wales. MMWR Morb Mortal Wkly Rep. 2005:54 Suppl:117-22. Available from: http://www.cdc.gov/mmwr/preview/ mmwrhtml/su5401a19.htm

18. Espino JU, Hogan WR, Wagner MM. Telephone triage: a timely data source for surveillance of influenza-like diseases. AMIA Annu Symp Proc. 2003:215-9.

19. Yih KW, Teates KS, Abrams A, Kleinman K, Pinner R, Harmon $R$, et al. Nurse call data for detection of influenza-like illness. Advances in Disease Surveillance. 2006;1:76. Available from: http://www.isdsjournal.org/article/viewArticle/122 
20. Yih WK, Teates KS, Abrams A, Kleinman K, Kulldorff M, Pinner R, et al. Telephone triage service data for detection of influenza-like illness. PLoS One. 2009;4(4):e526o.

21. Gault G, Larrieu S, Durand C, Josseran L, Jouves B, Filleul L. Performance of a syndromic system for influenza based on the activity of general practitioners, France. J Public Health (Oxf). 2009;31(2): 286-92.

22. Coory MD, Kelly H, Tippett V. Assessment of ambulance dispatch data for surveillance of influenza-like illness in Melbourne, Australia. Public Health. 2009;123(2):163-8.

23. Heffernan R, Mostashari F, Das D, Besculides M, Rodriguez C, Greenko J, et al. New York City syndromic surveillance systems. MMWR Morb Mortal Wkly Rep. 2004;53 Suppl:23-7. Available from: http://www.cdc.gov/mmwr/preview/mmwrhtml/ su5301a6.htm

24. Hripcsak G, Soulakis ND, Li L, Morrison FP, Lai AM, Friedman $C$, et al. Syndromic surveillance using ambulatory electronic health records. J Am Med Inform Assoc. 2009;16(3):354-61. 